\title{
Nucleation of a non-critical phase in a fluid near a critical point
}

\author{
Richard P. Sear \\ Department of Physics, University of Surrey \\ Guildford, Surrey GU2 7XH, United Kingdom \\ email: r.sear@surrey.ac.uk
}

October 28, 2018

\begin{abstract}
Phase diagrams of some globular proteins have a fluid-fluid transition as well as a fluid-crystal transition. Homogeneous nucleation of the crystal from the fluid phase near the critical point of the fluid-fluid transition is examined. As the fluid-fluid critical point is approached, the number of molecules in the critical nucleus, the nucleus at the top of the free energy barrier to nucleation, is found to diverge as the isothermal compressibility. This divergence is due to a layer of the fluid phase of width equal to the fluid's correlation length which surrounds the core of the nucleus; the number of molecules in a crystalline environment in the nucleus does not diverge. The free energy barrier to nucleation remains finite but its derivative with respect to the chemical potential is equal to minus the number of molecules in the critical nucleus and so diverges.
\end{abstract}

\section{Introduction}

The phase behaviour of a number of globular proteins has been studied and in addition to the fluid-to-crystal transition there is a metastable fluid-fluid transition, ${ }^{1,2}$ i.e., a transition between two fluid phases differing in density which ends at a critical point. This is analogous to the vapour-liquid transition of simple substances such as water. The transition is metastable: it exists within the fluid-crystal coexistence region. A fluid near the fluid-fluid critical point is anomalous, essentially because the correlation length is very large. Here we consider the free energy barrier $\Delta \Omega^{*}$ to crystallisation in a fluid which is close to the critical point. Crystallisation starts by the nucleation of a microscopic crystallite, this then grows to form a crystal. The rate at which such microscopic crystallites form is proportional to $\exp \left(-\Delta \Omega^{*} / k T\right)$ because their formation is an activated process. ${ }^{3,4}$ Here we study only the size and free energy of the critical nucleus, we leave consideration both of the dynamics of its formation and of its growth to later work. We also limit ourselves to temperatures above or equal to the critical temperature. Our main finding is that the number of molecules in the critical nucleus varies as $\chi_{T}$, the isothermal compressibility, near the critical point. As $\chi_{T}$ diverges at the critical point, so does the number of molecules in the nucleus. Within our theory the number of molecules in the critical nucleus is equal to minus the derivative of the free energy barrier with respect to the chemical potential and so near the critical point the barrier to nucleation is a rapidly decreasing function of chemical potential.

We focus on the universal aspects of critical nuclei near critical points. ${ }^{5}$ It is well-known that near a critical point fluids exhibit universal behaviour, behaviour which is determined solely by the universality class of the system, ${ }^{6}$ here that of the three-dimensional Ising model. Although in the centre of a critical nucleus the density will be far from the critical density, at the fringes of the nucleus far from the centre the density will be close to the bulk density of the fluid, and there in the fringes we find universal behaviour. We find that the fringe of the nucleus dominates the number of molecules in the nucleus but not the free energy. It does however dominate the derivative of the free energy with respect to the chemical potential as this is nothing but minus the number of molecules in the nucleus. In order to focus on the universal aspects we use a simple phenomenological theory and for simplicity we use a mean-field theory, although such theories have well-known deficiencies near critical points. ${ }^{6}$ We use a simple theory first used for droplets by Cahn and Hilliard. ${ }^{7}$ It is variously called Cahn-Hilliard theory, van der Waals-Cahn-Hilliard theory or the square gradient approximation. ${ }^{3,7-10}$

Numerical work on droplets near a critical point has been done by Talanquer and Oxtoby ${ }^{11}$ using a theory similar to a Cahn-Hilliard theory. This followed pioneering computer simulations of a critical nucleus near a critical point by ten Wolde and Frenkel. ${ }^{12}$ Talanquer and Oxtoby obtained nuclei with very large numbers of molecules near a critical point but did not perform the analytical analysis required to extract out the scaling near a critical point. Although their theory has an additional order parameter the scaling of the number of molecules near the critical point in their model is almost certainly the same as found here. See Refs. $13-15$ for other recent theoretical work on nucleation 
near a metastable transition. Also, more than twenty years ago, Widom ${ }^{9,16}$ used Cahn-Hilliard theory to look at the (planar) interface between coexisting phases, where one of these phases was at a critical point. He envisaged not a single component system with fluid-fluid and fluid-crystal phase transitions but a binary mixture with liquid-liquid demixing and vapour-liquid phase transitions. However, the universal aspects are the same for both systems. Thus, our calculations for the radial density profile of the nucleus are analogous to those for density profile of the planar interface found by Widom. In addition, our results are relevant to the nucleation of the vapour phase near the critical point of a metastable liquid-liquid demixing transition.

In the next section we derive analytic expressions, within a mean-field theory, for the density in the fringe of a nucleus in a near-critical fluid. Then we go on to derive an expression for the number of molecules, and hence the derivative of the free energy barrier near the critical point. The third and final section is a conclusion.

\section{Theory}

The critical nucleus is at the top of the barrier, it is at a maximum in the excess grand potential. The excess grand potential is the grand potential with a nucleus minus that without a nucleus. To start we require an expression for the excess grand potential of a nucleus $\Delta \Omega$ as a functional of the density function of the nucleus and then to find the density function which extremises this functional. For a crystalline nucleus, a rather complex functional is required, see for example the functional used to calculate the fluid-crystal interfacial tension for hard spheres. ${ }^{17}$ However, the universal effects we are looking for here occur at the fringe of the nucleus where the nucleus will have a density close to that of the fluid. There a much simpler functional suffices, such as those used to calculate fluid-fluid interfacial tensions and the excess grand potentials of fluid droplets in fluid phases.

For a spherically symmetric fluid droplet in a fluid, the density profile $\phi(r)$ is a function only of the distance $r$ from the centre of the droplet. The fluid phase has a density $\rho$ and a chemical potential $\mu ; \phi$ is the density at a point whereas $\rho$ is the bulk density of the fluid. Of course $\phi(r \rightarrow \infty)=\rho$. The standard Cahn-Hilliard expression for the grand potential cost $\Delta \Omega$ of such a droplet, as a functional of its density profile $\phi(r)$, is ${ }^{3,7-10}$

$$
\Delta \Omega=\int\left[\Delta \omega+\kappa(\nabla \phi)^{2}\right] \mathrm{d} \mathbf{r}
$$

where

$$
\Delta \omega(\phi)=f(\phi)-f(\rho)-\mu(\phi-\rho),
$$

is the work required per unit volume to change the density from $\rho$ to $\phi$, at a chemical potential $\mu$. $f(\phi)$ is the bulk Helmholtz free energy per unit volume of the fluid at a density $\phi$. The second term in Eq. (1) within the brackets is the gradient term: the grand potential cost due to variations in space of the density. The gradient squared term is the lowest order term in a gradient expansion and so is adequate when the density is slowly varying. The coefficient, $\kappa$ of this term is taken to be a constant. See Refs. 3,9,10 for its relation to the intermolecular potential.

The functional Eq. (1) will be totally inadequate within the crystalline core of a nucleus but we will not require either the density function or the contribution to the grand potential of this core. Thus, we will use Eq. (1) as our functional and end up with expressions which are the sum of two terms, one term from the core for which our expression will be wrong but which we will not evaluate, and another term from the fringe, for which Eq. (1) is a reasonable (mean-field) approximation which we will evaluate.

The critical nucleus is at the top of the free energy barrier and so is at a maximum of $\Delta \Omega$. Thus, for the critical nucleus we may set the functional derivative of $\Delta \Omega$ with respect to the density profile $\phi(r)$ to zero,

$$
\left(\frac{\partial \Delta \omega}{\partial \phi}\right)-2 \kappa \nabla^{2} \phi=0
$$

The curvature of the density profile at a point is proportional to the derivative of the excess grand potential with respect to the density at that point. Using Eq. (2) for $\Delta \omega$ in Eq. (3) we have

$$
\mu(\phi)-\mu-2 \kappa \nabla^{2} \phi=0
$$

where the $\mu$ without an argument is the chemical potential in the fluid, and $\mu(\phi)$ is the chemical potential of a bulk fluid at a density $\phi$. Once we have solved Eq. (4) we can insert the solution into Eq. (1) to obtain the excess grand potential of the critical nucleus, denoted by $\Delta \Omega^{*}$.

The excess number of molecules in the critical nucleus, $\Delta n^{*}$, is the number of molecules with the nucleus present minus the number without it. It may be obtained by integrating over the density profile,

$$
\Delta n^{*}=\int \Delta \phi(r) \mathrm{d} \mathbf{r}
$$


where $\Delta \phi(r)=\phi(r)-\rho$ is the excess density at a point, the density at a point minus the bulk density. The derivative of $\Delta \Omega^{*}$ with respect to the chemical potential of the fluid $\mu$ is, using Eq. (1),

$$
\left(\frac{\partial \Delta \Omega^{*}}{\partial \mu}\right)_{T}=-\int \Delta \phi(r) \mathrm{d} \mathbf{r}=-\Delta n^{*} .
$$

Thus, the derivative of $\Delta \Omega^{*}$ with respect to the chemical potential is simply $-\Delta n^{*}$. This result, that the derivative of the excess grand potential of the critical nucleus is minus the excess number of molecules in the nucleus, is often called the nucleation theorem. ${ }^{18-20}$

\subsection{The fringe of the nucleus}

Equation (4) can be solved numerically if the chemical potential is known as a function of density. Here, we would like to concentrate on the fringe of the critical nucleus. The fringe is the outermost part of the nucleus, where the density is near the density of the fluid, $\rho$. We define the fringe as being that part of the nucleus which is more than a distance $r_{c}$ from the centre. The distance $r_{c}$ is such that $\Delta \phi\left(r \geq r_{c}\right) / \rho \ll 1$. Fig. 3 is a schematic of the radial density profile of the nucleus. Now, if the fractional density difference $\Delta \phi / \rho \ll 1$ we can use a Taylor expansion for $\mu(\phi)-\mu$,

$$
\mu(\phi)-\mu=\Delta \phi\left(\frac{\partial \mu(\phi)}{\partial \phi}\right)_{\phi=\rho}+\cdots=\frac{\Delta \phi}{\rho^{2} \chi_{T}}+\cdots,
$$

where $\chi_{T}$ is the isothermal compressibility of the fluid (at a density $\rho$ ). The isothermal compressibility is defined $\operatorname{as}^{21}$

$$
\chi_{T}^{-1}=\rho^{2}\left(\frac{\partial \mu}{\partial \rho}\right)_{V, T} .
$$

Substituting Eq. (7) into Eq. (4) we have

$$
\frac{1}{\rho^{2} \chi_{T}} \Delta \phi(r)-2 \kappa \nabla^{2} \Delta \phi(r)=0 \quad \Delta \phi \ll \rho .
$$

This is the Helmholtz equation, for $\Delta \phi$, and the solution is a function of the Ornstein-Zernike form,

$$
\Delta \phi(r)=\rho \frac{\sigma}{r} \exp (-r / \xi),
$$

with $\xi$ the correlation length of the fluid, given by

$$
\xi^{2}=2 \kappa \rho^{2} \chi_{T}
$$

To obtain Eq. (10) the boundary conditions $\Delta \phi(r \rightarrow \infty) \rightarrow 0$ and $\Delta \phi\left(r_{c}\right)=\rho\left(\sigma / r_{c}\right) \exp \left(-r_{c} / \xi\right)$ were employed. $\sigma$ is a molecular length scale, a few nms for proteins. $\Delta \phi / \rho$ will, as required for Eq. (9), be small for $r \geq r_{c}$ provided that $r_{c}$ is a few times $\sigma$ or more. We do not need to specify $r_{c}$ beyond saying that it must be at least a few times $\sigma$. This implies a core a few molecules across, which is reasonable. From Eq. (10) we see that the width of the fringe is, as we might have expected, of the order of the correlation length $\xi$ in the surrounding fluid. This width will thus diverge as the fluid approaches a critical point.

\subsection{Near a critical point}

We now consider a nucleus in a fluid which is near a critical point, either at the critical density but just above the critical temperature, or at the critical temperature but at a density near the critical density. In either case as the critical point is approached the isothermal compressibility, and hence (see Eq. (11)) $\xi$, diverges. Within mean-field theory, along the critical isochore, i.e., with the density fixed at its value at the critical point $\rho_{c p}, \chi_{T}$ scales with temperature as ${ }^{3,6}$

$$
\chi_{T} \sim\left(T-T_{c p}\right)^{-1} \quad \rho=\rho_{c p} \quad T>T_{c p},
$$

where $T_{c p}$ is the temperature of the critical point. The compressibility diverges as one over the temperature difference to the critical point. An alternative path to the critical point is along the critical isotherm. We fix the temperature $T=T_{c p}$ and vary the density. Along the critical isotherm $\chi_{T}$ varies as

$$
\chi_{T} \sim\left(\rho-\rho_{c p}\right)^{-2} \quad T=T_{c p} .
$$


The compressibility diverges as one over the square of the density difference to the critical point.

Putting our solution for $\Delta \phi\left(r>r_{c}\right)$, Eq. (10), into Eq. (5) we find that when $\xi$ is very large and so the fringe correspondingly large in volume, that the fringe dominates $\Delta n^{*}$. Equation (5) is easily evaluated by substituting Eq. (10) for $\Delta \phi\left(r>r_{c}\right)$,

$$
\begin{aligned}
\Delta n^{*} & =\int_{r=0}^{r=r_{c}} \Delta \phi(r) \mathrm{d} \mathbf{r}+4 \pi \rho \sigma \xi^{2} \quad \xi \gg r_{c} \\
& =\int_{r=0}^{r=r_{c}} \Delta \phi(r) \mathrm{d} \mathbf{r}+8 \pi \sigma \kappa \rho^{3} \chi_{T} \quad \xi \gg r_{c},
\end{aligned}
$$

where we used the relation between $\xi$ and $\chi_{T}$, Eq. (11). The excess number of molecules $\Delta n^{*}$ is a sum of two terms. The first term, the integral in Eq. (14), comes from the core of the nucleus. It is of order $\left(\rho_{c}-\rho\right) r_{c}^{3}$ where $\rho_{c}$ is the density of the phase which is nucleating. It remains finite as the critical point is approached. However, the second term in Eq. (14), the contribution of the fringe to the number of molecules in the critical nucleus $\Delta n^{*}$, diverges. As the critical point is approached the number of molecules in the nucleus tends to infinity, as suggested by Talanquer and Oxtoby. ${ }^{11}$ It does so as the compressibility $\chi_{T}$. This is the central result of this work, and is a general result independent of the nature of the phase which is nucleating. The fringe of the nucleus is at densities close to the fluid's density and far from that in the core of the nucleus. So, the divergence in $\Delta n^{*}$ is from a divergent number of molecules at densities near the bulk fluid density: although they are part of a nucleus of a crystalline phase they themselves are in a fluid environment. The number of molecules which are in a crystalline environment does not diverge as the critical point is approached. Thus, within mean-field theory, along the critical isochore the number of molecules diverges as $\left(T-T_{c p}\right)^{-1}$ and along the critical isotherm as $\left(\rho-\rho_{c p}\right)^{-2}$.

Now we consider the contribution of the fringe to the grand potential of the critical nucleus. We require the Taylor expansion of $\Delta \omega$ about its value at the bulk density,

$$
\Delta \omega(\phi)=\frac{1}{2} \frac{(\Delta \phi)^{2}}{\rho^{2} \chi_{T}}+\cdots .
$$

The first nonzero term in the Taylor expansion of $\Delta \omega$ is the quadratic term as the first density derivative of $\Delta \omega$ is zero at $\rho$. Now, splitting the integration in Eq. (1) at $r_{c}$, and using the quadratic approximation, Eq. (15), for $\Delta \omega$ for $r>r_{c}$, we have

$$
\Delta \Omega=\int_{r=0}^{r=r_{c}}\left[\Delta \omega+\kappa(\nabla \phi)^{2}\right] \mathrm{d} \mathbf{r}+\int_{r=r_{c}}^{r=\infty}\left[\frac{1}{2} \frac{(\Delta \phi)^{2}}{\rho^{2} \chi_{T}}+\kappa(\nabla \Delta \phi)^{2}\right] \mathrm{d} \mathbf{r},
$$

where, for $r>r_{c}$, we also substituted $\Delta \phi$ for $\phi$ in the gradient term. After substituting Eq. (10) for $\Delta \phi$ in the second integral, we see that the contributions to the integral from both the terms in the integrand decreases as a function of radial distance $r$. Taking into account the factor of $r^{2}$ in $\mathrm{d} \mathbf{r}$ the integrand varies as $\exp (-2 r / \xi)$ times a quadratic polynomial in $1 / r$ : it is a monotonically decreasing function of $r$. The farther we go out into the fringe of the nucleus the smaller is the contribution to the excess grand potential, the barrier to nucleation. This means that the barrier to nucleation will be dominated by contributions from the core of the nucleus where the behaviour is non-universal and our approximations are invalid. Recall that our functional, Eq. (1), is a very poor approximation in the core and hence the first integral in Eq. (16) is not an accurate expression for the contribution of the core to the excess grand potential. We are thus unable to calculate the absolute excess grand potential of a critical nucleus near a critical point but we are of course able to calculate its derivative with respect to the chemical potential as this is just minus the excess number of molecules in the critical nucleus, which is dominated by the fringe.

\section{Conclusion}

Some globular proteins ${ }^{1,2}$ have two transitions: a fluid-crystal transition, call it transition $\alpha$, and a fluid-fluid transition, call it transition $\beta$. Transition $\alpha$ is always strongly first order but transition $\beta$ becomes continuous at the critical point. Here, we have studied homogeneous nucleation associated with transition $\alpha$ near a continuous transition $\beta$. Systems near a continuous transition show universal (within a universality class) behaviour and are very susceptible to a perturbation - response functions such as the compressibility take large values. The nucleus associated with homogeneous nucleation of a transition $\alpha$ therefore perturbs the highly susceptible fluid near transition $\beta$. Because the fluid is near this continuous transition, the response functions such as the isothermal compressibility $\chi_{T}$, are large. The perturbation due to the nucleus is therefore large, it extends out to the large distance $\xi$ and involves a number of molecules which scales with $\chi_{T}$. The free energy barrier to homogeneous nucleation of transition $\alpha$ is then a rapidly decreasing function of chemical potential. As suggested by ten Wolde and Frenkel, ${ }^{12}$ the continuous transition $\beta$ helps the dynamics of transition $\alpha$. This is true in general, not just for crystallisation near a critical point. The nucleation of the vapour phase when a 
mixture of liquids boils near a metastable critical point of liquid-liquid demixing is another example. Note that there $\Delta n^{*}$ is negative and will tend towards $-\infty$ not $+\infty$.

The theory of section 2 is a simple mean-field theory. However, our conclusion that $\Delta n^{*} \sim \chi_{T}$ near the critical point may be valid beyond mean-field theory. If so then the divergence of $\Delta n^{*}$ along a particular path in the diagram, e.g., the critical isochore, will occur with whatever is the correct critical exponent of $\chi_{T}$ along that path. For example, for nucleation near a critical point in the universality class of the Ising model in three dimensions, $\chi_{T}$ scales as $\left(T-T_{c p}\right)^{-1.24}$ along the critical isochore not $\left(T-T_{c p}\right)^{-1}$ as mean-field theory predicts, see Refs. 3,6 .

The starting point for most theoretical studies of nucleation is classical nucleation theory. ${ }^{3,4}$ Within classical nucleation theory the critical nucleus is modeled as a small crystallite, assumed to have bulk properties, separated from the surrounding metastable fluid by an interface, assumed to have the same surface tension as that between the coexisting bulk phases and to be thin, no more than a couple of molecules thick. For a nucleus in a fluid far from a fluid-fluid critical point, this assumption of a thin interface with the bulk surface tension is not unreasonable. The correlation lengths in the fluid and crystalline phases will both be small and so will the width of the interface. Then if the width of the interface is small with respect to the diameter of the nucleus then approximating its surface tension by that of the planar interface between coexisting phases is reasonable. However, for a nucleus in a near-critical fluid, the correlation length in the surrounding fluid $\xi$ may be very large. So, for a crystal nucleus which is only a few lattice spacings across the interface between it and the surrounding fluid may be much thicker than the diameter of the crystalline part of the nucleus. Then this interface will be very different from that assumed within classical nucleation theory, and so classical nucleation theory will yield only a poor estimate of the barrier to nucleation.

It is a pleasure to thank R. Bowles for useful discussions. Work supported by EPSRC (GR/N36981).

\section{References}

[1] M. L. Broide, C. R. Berland, J. Pande, O. O. Ogun and G. B. Benedek, Proc. Nat. Acad. Sci. 88, 5660 (1991).

[2] M. Muschol and F. Rosenberger, J. Chem. Phys. 107, 1953 (1997).

[3] P. G. Debenedetti, Metastable Liquids (Princeton University Press, Princeton, 1996).

[4] J. D. Gunton, M. San Miguel and P. S. Sahni, in Phase Transitions volume 8, edited by C. Domb and J. L. Lebowitz (Academic Press, London, 1983).

[5] Note that conventionally 'critical' is used to denote both a point in the phase diagram and the crystallite at the top of the barrier although there is no connection between the two uses of the word. Although this terminology is a little unfortunate we will use it here and so we will be studying a critical nucleus near a critical point.

[6] P. M. Chaikin and T. C. Lubensky, Principles of Condensed Matter Physics (Cambridge University Press, Cambridge, 1995).

[7] J. W. Cahn and J. E. Hilliard, J. Chem. Phys. 31, 688 (1959).

[8] J. W. Cahn and J. E. Hilliard, J. Chem. Phys. 28, 258 (1958).

[9] B. Widom, Chem. Soc. Rev. 14, 121 (1985).

[10] R. Evans, Les Houches, Session XLVIII: Liquids at Interfaces, edited by J. Charvolin, J. F. Joanny and J. ZinnJustin (Elsevier, Amsterdam, 1989)

[11] V. Talanquer and D. W. Oxtoby, J. Chem. Phys. 109, 223 (1998).

[12] P. R. ten Wolde and D. Frenkel, Science 277, 1975 (1997).

[13] C. Haas and J. Drenth, J. Phys. Chem. B 104, 368 (2000).

[14] N. M. Dixit and C. F. Zukoski, J. Coll. Int. Sci. 228, 359 (2000).

[15] R. P. Sear, cond-mat/9912199 (http://xxx.lanl.gov).

[16] B. Widom, J. Chem. Phys. 67, 872 (1977).

[17] R. Ohnesorge, H. Löwen and H. Wagner, Phys. Rev. E 50, 4801 (1994).

[18] D. Kashchiev, J. Chem. Phys. 76, 5098 (1982). 
Figure 1: A schematic of the radial density profile of a droplet: $\phi(r)$ is the density at a distance $r$ from the centre of the droplet. The density of the surrounding fluid is $\rho$, and $r_{c}$ is the (somewhat arbitrary) point where we divide the density profile into a central part and an outer part.

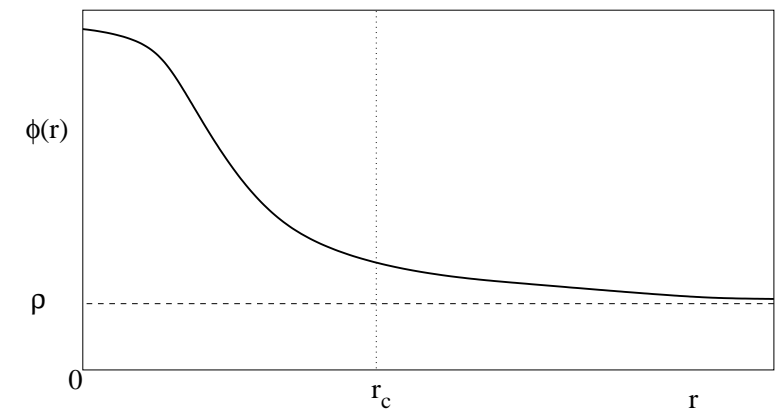

[19] Y. Viisanen, R. Strey and H. Reiss, J. Chem. Phys. 99, 4680 (1993).

[20] R. K. Bowles, R. McGraw, P. Schaaf, B. Senger, J.-C. Voegel and H. Reiss, J. Chem. Phys. 113, 4524 (2000).

[21] J.-P. Hansen, and I. R. McDonald, Theory of Simple Liquids (Academic Press, London, 2nd Edition, 1986). 